\title{
Dentate Circuitry as a Model to Study Epileptogenesis
}

\author{
Ryuta Koyama \\ Laboratory of Chemical Pharmacology, Graduate School of Pharmaceutical Sciences, The University \\ of Tokyo; 7-3-1 Hongo, Bunkyo-ku, Tokyo 113-0033, Japan. \\ Received February 8, 2016
}

\begin{abstract}
Epileptogenesis, which can be initiated by brain insults or gene mutations in the normal brain, is defined as the gradual (months to years) process of epilepsy development that begins before the first epileptic seizure. Epileptogenic changes include induction of immediate early genes, post-translational modification of ionchannel functions, neuronal death, gliosis, and reorganization of neural circuits. Each of these changes alone or in combination can contribute to an epileptogenic focus, which is defined by the minimal cortical region that is necessary and sufficient to induce synchronized epileptic bursting activity in neurons. Therefore to discover and develop anti-epileptogenic drugs it is essential to unveil the cellular and molecular mechanisms underlying the development of epileptogenic foci. Among the epileptogenic changes, abnormally appended excitatory recurrent circuits can directly cause synchronized bursting of neuron activity. Here, I will introduce and discuss the mechanisms underlying the development of two representative abnormal neural circuits, namely, hippocampal mossy fiber sprouting and ectopic granule cells, which are found in the dentate gyrus of patients with mesial temporal lobe epilepsy and its animal models.
\end{abstract}

Key words epilepsy; epileptogenesis; dentate gyrus; febrile seizure; ectopic granule cell; mossy fiber sprouting

\section{INTRODUCTION}

To understand the structural and functional basis of abnormal neural circuits in the epileptic dentate gyrus, I briefly introduce the anatomical properties of the dentate gyrus (readers are recommended to read Amaral et al. ${ }^{1)}$ for further information). The dentate gyrus is an archicortical region in the limbic system that comprises a part of the hippocampal formation, along with the hippocampus. The dentate gyrus exhibits similar anatomical structures throughout the septotemporal axis and thus is not divided into subregions. However, it should be noted that the frequency of epileptogenic neural circuits could differ along the septotemporal axis. In the transverse surface that is vertical to the septotemporal axis, the dentate gyrus contains three layers: the molecular layer, granule cell layer, and polymorphic layer, which is often referred to as the hilus. The basic three-layered structure of the dentate gyrus is common across all species in which it has been studied, including rodents and primates.

A simple schematic diagram of neural circuits in the normal and epileptic dentate gyrus is shown in Fig. 1. The molecular layer is a relatively cell-free layer and is occupied by the characteristic besom-shaped dendrites of granule cells. The dendrites of granule cells receive excitatory synaptic contacts from the fibers of the perforant path that originates in the entorhinal cortex. The granule cell layer is densely packed with dentate granule cells. Although the granule cell layer mainly contains dentate granule cells, there is a sparse distribution of glial cells and their fibers and interneurons that are located at the boundary of the granule and polymorphic layers. The axons of granule cells, i.e., the hippocampal mossy fibers, exhibit branching collaterals in the polymorphic layer and provide a number of excitatory synaptic connections with a variety of inhibitory interneurons in the dentate gyrus, including the dentate pyramidal basket cells. The main shaft of mossy fibers projects further toward the CA3 field of the hippocampus. The mossy fibers mainly provide excitatory synaptic contacts to the dendrites of CA3 pyramidal cells, relaying information from the entorhinal cortex to CA3. It is notable that CA3 pyramidal cells provide excitatory synaptic connections with not only CA1 pyramidal cells but also several interneurons located in the polymorphic layer. Finally, the polymorphic layer contains a variety of interneurons, including excitatory interneurons, namely, the mossy cell. The mossy cell receives excitatory synaptic contacts from mossy fibers and provides excitatory synaptic contacts to pyramidal basket cells. Thus the excitatory inputs from mossy cells to pyramidal basket cells eventually mediate an inhibitory feedback circuitry to dentate granule cells.

It has been suggested that the dentate gyrus is engaged in epileptogenesis in mesial temporal lobe epilepsy, in which the hippocampal formation plays a pivotal role as an epileptic focus because the function of the dentate gyrus as a highresistance gate is compromised under the epileptic condition, ${ }^{2,3)}$ allowing the invasion of epileptiform activity from the entorhinal cortex to the hippocampus. The "failsafe-like" property of the dentate safety gate results from the inhibitory feedback circuit to dentate granule cells and the static characteristics of dentate granule cells, which have a hyperpolarized resting membrane potential, high $\mathrm{K}^{+}$conductance, low $\mathrm{Ca}^{2+}$ conductance, and strong tonic and phasic $\gamma$-aminobutyric acid (GABA) inhibition and lack the intrinsic capacity for burst discharges. ${ }^{4)}$ In the epileptic dentate gyrus, the excitatory recurrent circuits and loss of various inhibitory interneurons in the polymorphic layer amplify the epileptic bursting of neurons, transforming the dentate gyrus from a safety gate to an amplifier for epileptic activity invading from the entorhinal cortex. However, it should be noted that the term epileptogenesis indicates the process before the onset of spontaneous seizures and not the chronic or aggravation process of epilepsy. 

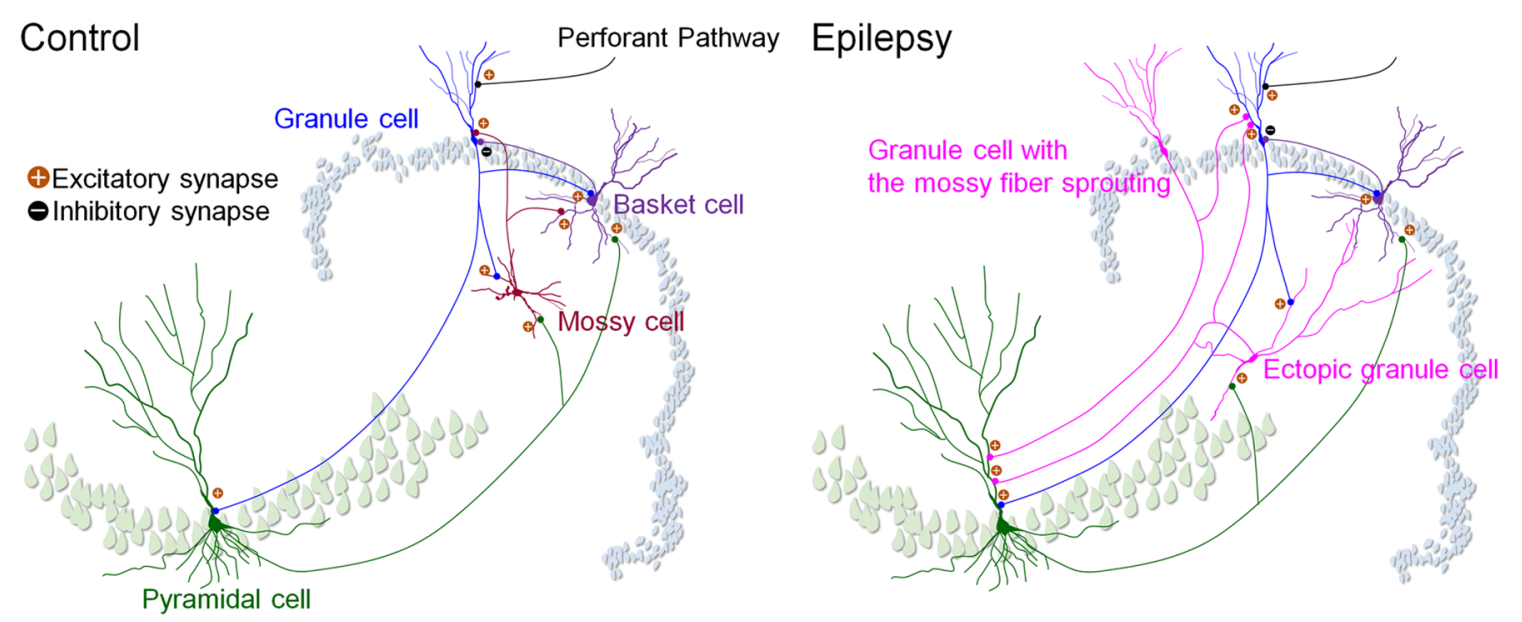

Fig. 1. Anatomy of Dentate Circuits in Control and Epileptic Conditions

In the epileptic dentate gyrus (right), mossy fiber sprouting and ectopic granule cells add recurrent excitatory circuits.

Thus it is crucial carefully to determine whether abnormal neural circuits in the dentate gyrus emerge before the onset of spontaneous seizures.

\section{MOSSY FIBER SPROUTING HYPOTHESIS}

Hippocampal mossy fibers, which are axons of dentate granule cells, normally project to the CA3 field of the hippocampus. Mossy fibers sometimes project through the granule cell layer, but they are scarcely found in the inner molecular layer in normal conditions. However, in the dentate gyrus of patients with mesial temporal lobe epilepsy and the corresponding animal models, mossy fibers exhibit robust branching in the polymorphic layer. These branching collaterals frequently project to the inner molecular layer and mainly provide excitatory synapses to granule cell dendrites. This reorganization of the dentate neural circuit is called mossy fiber sprouting. To study the details of mossy fiber sprouting, readers are recommended to see the review articles published by Nadler, ${ }^{4)}$ Koyama and Ikegaya,${ }^{5}$ and Sloviter et al. ${ }^{6)}$
Elegant electron microscope studies have revealed that sprouted mossy fibers make mostly asymmetric excitatory synapses on the dendritic spines of granule cells in rat models of temporal lobe epilepsy. ${ }^{7,8)}$ Furthermore, stimulation of the performant pathway induces epileptiform activity in the granule cell layer in slices prepared from patients with temporal lobe epilepsy ${ }^{9,10)}$ and its animal models. ${ }^{11)}$ Consistent with these findings, several studies reported a correlation between the development of mossy fiber sprouting and a progression of the frequency and severity of behavioral seizures. ${ }^{11-13)}$ These studies form the basis of the mossy fiber sprouting hypothesis, which indicates that sprouted mossy fibers reorganize the dentate neural circuits into epileptic, recurrent excitatory circuits.

For the cellular and molecular mechanisms underlying mossy fiber sprouting, we hypothesized the 3 -step abnormal axon guidance model. ${ }^{5)}$ In step 1 , the branching of mossy fibers in the hilus is triggered by hyperexcitability in the dentate neural circuits. In step 2, an aberrant guidance of mossy fibers leads these axons to the inner molecular layer instead of their normal target, CA3. In step 3, the abnormally sprouted

Dr. Koyama attended the University of Tokyo, Faculty of Pharmaceutical Sciences, in 2000 with a concentration in neuroscience and pharmacology. Following his undergraduate work, he attended the University of Tokyo, Graduate School of Pharmaceutical Sciences, Laboratory of Chemical Pharmacology (Dr. Norio Matsuki laboratory). His focus in graduate school was on the axon guidance mechanism of hippocampal mossy fibers. Specifically, he investigated the cellular and molecular mechanisms that induce abnormal sprouting of the hippocampal mossy fibers in epilepsy. Soon after obtaining a Ph.D. in Pharmaceutical Sciences in 2006, he became an Associate Professor at the University of Tokyo, Graduate School of Pharmaceutical Sciences, Laboratory of Chemical Pharmacology (Dr. Norio Matsuki laboratory), and expanded his research on abnormal neural circuit formation in the epileptic brain, finding that the diuretic bumetanide can block the abnormal neural circuit

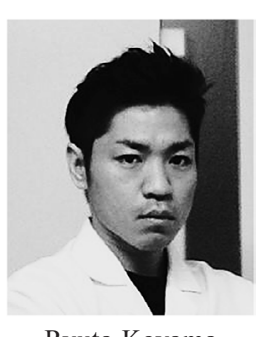

Ryuta Koyama formation during childhood and the development of epilepsy (Koyama et al., Nature Medicine, 2012). In 2010, he attended Harvard Medical School, Boston Children's Hospital, F.M. Kirby Neurobiology Research Center, as a postdoctoral researcher in the laboratory of Dr. Beth Stevens. In Dr. Stevens laboratory, he investigated the role of neuronal activity and microglia in neural circuit formation. After coming back to the University of Tokyo in 2013, he continued his interest in neuron-microglia interaction in health and disease. In 2015, he promoted to an Associate Professor at the University of Tokyo, Graduate School of Pharmaceutical Sciences, Laboratory of Chemical Pharmacology (Dr. Yuji Ikegaya laboratory). He is now investigating the cellular and molecular mechanisms how microglia detect the environmental changes and react to them in the brain. He is also interested in the mechanisms how microglia reorganize adult neural circuits both in health and disease. 
mossy fibers are maintained. Using a combination of in vivo and slice culture studies, we first determined that normal axon guidance of mossy fibers to CA3 is supported by activation of group II metabotropic glutamate receptors ${ }^{14)}$ and both diffusible and contact-dependent axon guidance cues. ${ }^{15)}$ Next, we found that activity-dependent expression of brain-derived neurotrophic factor (BDNF) induces robust branching of mossy fibers in the dentate hilus through activation of tropomyosin receptor kinase B (TrkB) (step 1). ${ }^{16,17)}$ We recently revealed that activity-dependent increases in intracellular cAMP levels alone could induce mossy fiber branching using an optogenetic method to photo-activate primary cultures of granule cells expressing photoactivated adenylyl cyclase (PAC), which produces cAMP in response to blue-light exposure. ${ }^{18)}$ For the mechanisms underlying step 2, we found that disruption of Netrin-1-dependent axon guidance of mossy fibers results in aberrant projection of these axons toward the inner molecular layer. Specifically, an activity-dependent increase in intracellular cAMP levels led to an imbalance in expression levels of deleted in colorectal cancer (DCC) and uncoordinated-5A (UNC5A) receptors, both of which are receptors of Netrin-1 and mediate attractant and repellent responses, respectively, of mossy fiber growth cones (step 2). ${ }^{19)}$ In addition, we found that repulsive guidance molecule a (RGMa), which is expressed in the granule cell layer, blocks activity-dependent mossy fiber sprouting in cultured hippocampal slices, probably by preventing mossy fibers from invading the inner molecular layer. ${ }^{20)}$ Finally, for step 3, AMP-activated protein kinase (AMPK) mediates activity-dependent formation and maintenance of mossy fiber branching by recruiting mitochondria to the axon. ${ }^{21)}$ Thus the above molecules are potential targets for preventing mossy fiber sprouting in the epileptic brain.

It should be noted, however, that sprouted mossy fibers could also provide synaptic connections to dentate pyramidal basket cells, one of the major inhibitory interneurons. This phenomenon is the basis for the recurrent inhibitory hypothesis. $\left.{ }^{6}\right)$ According to this hypothesis, loss of mossy cells after seizures results in a lack of mossy cell-basket cell connections, which then leads to transient excitation of granule cells and mossy fiber sprouting. Eventually, the transient excitation is overcome by newly formed inhibitory feedback circuits consisting of excitatory inputs from sprouted mossy fibers to basket cells.

In addition, granule cells are thought to release glutamate and the inhibitory neurotransmitter GABA under specific conditions, ${ }^{22)}$ and expression levels of GABA and its synthesizing enzyme glutamic acid decarboxylase 67 (GAD67) are increased after epileptic seizures. ${ }^{23,24)}$ Thus it is also interesting to hypothesize that sprouted mossy fibers release GABA and directly suppress synchronized activity of granule cells in the epileptic dentate gyrus. However, no direct evidence has been reported so far to explain such defense mechanisms. Further, several reports suggested that excitatory GABA signaling shaped by a disrupted $\mathrm{Cl}^{-}$homeostasis could induce epileptic bursting of neurons in specimens prepared from patients with temporal lobe epilepsy. ${ }^{25}$ Therefore allowing that GABA is released from sprouted mossy fibers, whether GABA increases or decreases the excitability of dentate neural circuits remains to be clarified by future studies.

Finally, several studies have suggested that there is no correlation between the sprouting of mossy fibers and the pro- gression of epileptogenesis. For example, it has been reported that the first appearance of spontaneous seizures precedes mossy fiber sprouting in a status epilepticus model of epilepsy. ${ }^{26}$ ) These findings indicate that mossy fiber sprouting is a consequence of seizures. Thus it is likely that mossy fiber sprouting contributes to the chronicity and aggravation of epilepsy rather than epileptogenesis.

\section{ECTOPIC GRANULE CELL HYPOTHESIS}

An abnormal location of granule cells was reported in adult patients with temporal lobe epilepsy and the corresponding animal models. ${ }^{27-31)}$ In these reports, the somata of granule cells were dispersed, forming a wider granule cell layer than normal (granule cell dispersion), ${ }^{27,28)}$ whereas ectopically located granule cells were found in the dentate hilus (ectopic granule cells). ${ }^{31)}$ We also found that hilar ectopic granule cells are induced by early-childhood seizures in mice. ${ }^{32)} \mathrm{Al}$ though hilar ectopic granule cells are sometimes found under physiological (non-epileptic) conditions, the density of ectopic granule cells significantly increases in temporal lobe epilepsy. The ectopic granule cells exhibit almost equally spaced localization along the septal axis, and sometimes exist as a cluster along the temporal axis. Most ectopic granule cells are localized in the hilus and absent from the CA3 field of the hippocampus. ${ }^{33)}$ Ectopic granule cells often possess bipolar dendrites receiving excitatory inputs from normal granule cells in the granule cell layer and also from CA3 pyramidal cells. Ectopic granule cells project their axons to the molecular layer in addition to CA3 under epileptic conditions. The excitatory loops made by ectopic granule cells are suggested to induce hyperexcitability of the hippocampus, leading to epileptogenesis. Indeed, ectopic granule cells produce spontaneous and rhythmic burst firing synchronized with epileptic burst firing of CA3 pyramidal cells. ${ }^{34)}$ Furthermore, cognitive impairment, particularly memory disruption, is a considerable complication in temporal lobe epilepsy, ${ }^{35)}$ and ectopic cells have been suggested to disrupt dentate gyrus-dependent learning and memory. ${ }^{36,37)}$

We found that ectopic granule cells contribute to the development of epilepsy in a rat model of complex febrile seizures. $^{38,39)}$ Febrile seizures are the most common convulsive events in early childhood between the ages of 6 months and 5 years, and some studies report that prolonged complex febrile seizures are subsequently associated with the development of temporal lobe epilepsy. ${ }^{40)}$ During the early postnatal period, when the prevalence of febrile seizures is highest, the newborn granule cells robustly proliferate in the deep hilus near the CA3c field and migrate toward the granule cell layer. ${ }^{32,41)}$ This migration is probably mediated via polysialic acid (PSA), which is present in the granule surface to modulate interactions among granule cells. ${ }^{42}$ ) We found that the migration of newborn granule cells is attenuated by febrile seizures, and these cells mature into ectopic granule cells that persist into adulthood. ${ }^{38)}$ Specifically, febrile seizures induce upregulation of $\mathrm{GABA}_{\mathrm{A}}-\mathrm{Rs}$ in migrating immature granule cells, and excitatory GABA signaling causes a reversal in the direction of granule cell migration via abnormal $\mathrm{Ca}^{2+}$-influx into the leading processes. We also determined that the emergence of ectopic granule cells precedes the onset of the first spontaneous seizure. In addition, there was a significant correlation 
between the number of ectopic granule cells and the frequency of seizures. As for the cellular and molecular mechanisms underlying febrile seizure-induced deficits in cell migration, we identified enhanced excitatory GABAergic inputs to the migrating granule cells. Finally, abnormal migration and the development of epilepsy were prevented by pharmacologically inhibiting $\mathrm{Na}^{+} \mathrm{K}^{+} 2 \mathrm{Cl}^{-}$co-transporter isoform 1 (NKCC1), which shapes the excitatory action of GABA, using the diuretic bumetanide. These results suggest that bumetanide serves as an anti-epileptogenic drug by preventing the formation of abnormal neural circuits in the dentate gyrus.

\section{BUMETANIDE AS POTENTIAL ANTI-EPILEPTO- GENIC DRUG}

One major clinical issue is that commonly used GABAmimetic and GABA-modulating anti-epileptic drugs are not necessarily efficacious against early-childhood seizures because GABA can be excitatory in the developing brain, which contains many immature neurons. ${ }^{43)}$ The neurotransmitter GABA acts on $\mathrm{Cl}^{-}$-permeable $\mathrm{GABA}_{\mathrm{A}}$ receptors $\left(\mathrm{GABA}_{\mathrm{A}}{ }^{-}\right.$ Rs). Whether GABA provokes inhibitory or excitatory actions depends on intracellular $\mathrm{Cl}^{-}$levels and the $\mathrm{Cl}^{-}$equilibrium potential, which are mainly controlled by cation-chloride cotransporters (CCCs). ${ }^{44)}$ Two members of the CCC family, $\mathrm{NKCC1}$, which mediates $\mathrm{Cl}^{-}$influx, and $\mathrm{K}^{+} 2 \mathrm{Cl}^{-}$cotransporter isoform 2 (KCC2), which mediates $\mathrm{Cl}^{-}$efflux, play important roles in regulating the developmental changes in the actions of GABA. Early expression of $\mathrm{NKCC1}$ and late expression of $\mathrm{KCC} 2$ underlie the excitatory action of GABA in immature neurons because of an elevated intracellular $\mathrm{Cl}^{-}$level and a depolarized $\mathrm{Cl}^{-}$equilibrium potential. In mature neurons, KCC2 predominates and reduces the intracellular $\mathrm{Cl}^{-}$level, shaping the inhibitory action of GABA. Interestingly, a portion of neurons in adult patients with epilepsy express high levels of $\mathrm{NKCC} 1$ and low levels of $\mathrm{KCC} 2$ similar to those of immature neurons, resulting in excitatory GABA actions in these neurons. ${ }^{25)}$

To date, experimental and clinical studies suggest that the diuretic bumetanide, which is a selective inhibitor of $\mathrm{NKCC1}$, could be useful for the treatment of epilepsy. ${ }^{45,46)}$ Dzhala et $a l .{ }^{47)}$ reported that bumetanide blocks kainic acid-induced seizures in neonatal rats whereas Brandt et al. ${ }^{48)}$ reported that bumetanide in combination with phenobarbital exerts diseasemodifying effects in the rat pilocarpine model of temporal lobe epilepsy. In addition, bumetanide was reported to reduce seizure frequency in patients with temporal lobe epilepsy. ${ }^{49)}$ It should be noted, however, that the use of bumetanide can induce hypokalemic alkalosis, which may promote seizures, ${ }^{45,46}$ ) and can attenuate normal development of the brain because GABA is a major source of excitation that exerts trophic actions in immature neurons. ${ }^{50)}$ Another issue to be solved is how to deliver highly ionized bumetanide into the brain. Future studies are required to solve the above issues to consider bumetanide as a novel anti-epileptogenic drug.

\section{CONCLUSION}

Both mossy fiber sprouting and ectopic granule cells add excitatory recurrent circuits in the epileptic dentate gyrus. Specifically, these recurrent circuits provide excitatory syn- apses in the inner molecular layer, probably inhibiting the function of the dentate safety gate, which blocks invasion of epileptic activity from the entorhinal cortex. However, it is also possible that newly added excitatory synapses enhance feedback inhibition of the granule cells via the dentate basket cells. Thus future studies are required to determine whether and how mossy fiber sprouting and ectopic granule cells in combination affect the excitatory versus inhibitory balance (E/I balance) in the epileptic dentate gyrus.

Anti-epileptic drugs to date have been used mainly for palliative treatment to suppress pre-existing epileptic seizures but not the first spontaneous seizure. Thus these drugs can be referred to as "anti-epileptic seizure" drugs. If we were to continue drug screenings using only a few different animal models of epilepsy, we will not overcome the current situation in which seizures in $20-30 \%$ of patients cannot be controlled by anti-epileptic drugs. ${ }^{51,52)}$ One major reason for this issue is that drug development that depends on logical steps has been considered relatively less serious and thus the cellular and molecular mechanisms of epilepsy remain elusive. Truly to understand and overcome epilepsy, and to open a door for the development of anti-epileptogenic drugs, we need to clarify the cellular and molecular mechanisms underlying epileptogenesis step by step, including the mechanisms that induce abnormal neural circuit formation. For this purpose, the structural and functional changes of the dentate gyrus under epileptic conditions can serve as a good model. However, it should be noted that several modifications take place in both excitatory and inhibitory networks in the epileptic dentate gyrus. Thus we should pay attention to the relations between a comprehensive dentate circuit function and epileptogenesis.

The reality of the failure to control seizures in $20-30 \%$ of patients with epilepsy with existing anti-epileptic drugs simultaneously implies the existence of undiscovered drug targets. For example, the neuron-glia interaction definitively affects the E/I balance of neural circuits in the epileptic brain. ${ }^{53)}$ Glial cells can modify network excitability in the epileptic brain functionally by regulating the concentrations of neurotransmitters and ions at the synaptic cleft as well as structurally by eliminating synapses and neurons by their phagocytic capacity. In this way, research of epileptogenesis will bring significant benefits for not only the development of novel drugs but also the biological findings regarding neural circuit formation.

Finally, precise biomarkers that determine whether antiepileptogenic drugs should be administered to patients are necessary. The epileptogenic process frequently takes place during development; therefore prediction and preparation for unknown adverse effects are necessary for the successful development of anti-epileptogenic drugs.

Acknowledgments This work was supported by JSPS KAKENHI Grant Numbers 26460094 and 26117504.

Conflict of Interest The author declares no conflict of interest.

\section{REFERENCES}

1) Amaral DG, Scharfman HE, Lavenex P. The dentate gyrus: fundamental neuroanatomical organization (dentate gyrus for dummies). Prog. Brain Res., 163, 3-22, 788-790 (2007). 
2) Heinemann U, Beck H, Dreier JP, Ficker E, Stabel J, Zhang CL. The dentate gyrus as a regulated gate for the propagation of epileptiform activity. Epilepsy Res. Suppl., 7, 273-280 (1992).

3) Lothman EW, Stringer JL, Bertram EH. The dentate gyrus as a control point for seizures in the hippocampus and beyond. Epilepsy Res. Suppl., 7, 301-313 (1992).

4) Nadler JV. The recurrent mossy fiber pathway of the epileptic brain. Neurochem. Res., 28, 1649-1658 (2003).

5) Koyama R, Ikegaya Y. Mossy fiber sprouting as a potential therapeutic target for epilepsy. Curr. Neurovasc. Res., 1, 3-10 (2004).

6) Sloviter RS, Bumanglag AV, Schwarcz R, Frotscher M. Abnormal dentate gyrus network circuitry in temporal lobe epilepsy. Jasper's Basic Mechanisms of the Epilepsies [Internet]. 4th ed. (Noebels JL, Avoli M, Rogawski MA, Olsen RW, Delgado-Escueta AV eds.), National Center for Biotechnology Information U.S.A. (2012).

7) Buckmaster PS, Zhang GF, Yamawaki R. Axon sprouting in a model of temporal lobe epilepsy creates a predominantly excitatory feedback circuit. J. Neurosci., 22, 6650-6658 (2002).

8) Cavazos JE, Zhang P, Qazi R, Sutula TP. Ultrastructural features of sprouted mossy fiber synapses in kindled and kainic acid-treated rats. J. Comp. Neurol., 458, 272-292 (2003).

9) Masukawa LM, Higashima M, Kim JH, Spencer DD. Epileptiform discharges evoked in hippocampal brain slices from epileptic patients. Brain Res., 493, 168-174 (1989).

10) Isokawa M, Fried I. Extracellular slow negative transient in the dentate gyrus of human epileptic hippocampus in vitro. Neuroscience, 72, 31-37 (1996).

11) Patrylo PR, Schweitzer JS, Dudek FE. Abnormal responses to perforant path stimulation in the dentate gyrus of slices from rats with kainate-induced epilepsy and mossy fiber reorganization. Epilepsy Res., 36, 31-42 (1999).

12) Sutula T, He XX, Cavazos J, Scott G. Synaptic reorganization in the hippocampus induced by abnormal functional activity. Science, 239, 1147-1150 (1988).

13) Cavazos JE, Golarai G, Sutula TP. Mossy fiber synaptic reorganization induced by kindling: time course of development, progression, and permanence. J. Neurosci., 11, 2795-2803 (1991).

14) Koyama R, Yamada MK, Nishiyama N, Matsuki N, Ikegaya Y. Group II metabotropic glutamate receptor activation is required for normal hippocampal mossy fibre development in the rat. J. Physiol., 539, 157-162 (2002).

15) Koyama R, Yamada MK, Nishiyama N, Matsuki N, Ikegaya Y. Developmental switch in axon guidance modes of hippocampal mossy fibers in vitro. Dev. Biol., 267, 29-42 (2004a).

16) Koyama R, Yamada MK, Fujisawa S, Katoh-Semba R, Matsuki $\mathrm{N}$, Ikegaya $\mathrm{Y}$. Brain-derived neurotrophic factor induces hyperexcitable reentrant circuits in the dentate gyrus. J. Neurosci., 24, 7215-7224 (2004b)

17) Koyama R, Ikegaya Y. To BDNF or not to BDNF: that is the epileptic hippocampus. Neuroscientist, 11, 282-287 (2005).

18) Zhou Z, Tanaka KF, Matsunaga S, Iseki M, Watanabe M, Matsuki N, Ikegaya Y, Koyama R. Photoactivated adenylyl cyclase (PAC) reveals novel mechanisms underlying cAMP-dependent axonal morphogenesis. Sci. Rep., 5, 19679 (2016).

19) Muramatsu R, Nakahara S, Ichikawa J, Watanabe K, Matsuki N, Koyama R. The ratio of 'deleted in colorectal cancer' to 'uncoordinated-5A' netrin-1 receptors on the growth cone regulates mossy fibre directionality. Brain, 133, 60-75 (2010).

20) Shibata K, Nakahara S, Shimizu E, Yamashita T, Matsuki N, Koyama R. Repulsive guidance molecule a regulates hippocampal mossy fiber branching in vitro. Neuroreport, 24, 609-615 (2013).

21) Tao K, Matsuki N, Koyama R. AMP-activated protein kinase mediates activity-dependent axon branching by recruiting mitochondria to axon. Dev. Neurobiol., 74, 557-573 (2014).

22) Münster-Wandowski A, Gomez-Lira G, Gutierrez R. Mixed neurotransmission in the hippocampal mossy fibers. Front. Cell. Neuro- sci., 7, 210 (2013).

23) Ramírez M, Gutierrez R. Activity-dependent expression of GAD67 in the granule cells of the rat hippocampus. Brain Res., 917, 139$146(2001)$.

24) Gómez-Lira G, Trillo E, Ramirez M, Asai M, Sitges M, Gutierrez R. The expression of GABA in mossy fiber synaptosomes coincides with the seizure-induced expression of GABAergic transmission in the mossy fiber synapse. Exp. Neurol., 177, 276-283 (2002).

25) Cohen I, Navarro V, Clemenceau S, Baulac M, Miles R. On the origin of interictal activity in human temporal lobe epilepsy in vitro. Science, 298, 1418-1421 (2002).

26) Nissinen J, Lukasiuk K, Pitkanen A. Is mossy fiber sprouting present at the time of the first spontaneous seizures in rat experimental temporal lobe epilepsy? Hippocampus, 11, 299-310 (2001).

27) Houser CR. Granule cell dispersion in the dentate gyrus of humans with temporal lobe epilepsy. Brain Res., 535, 195-204 (1990).

28) Lurton D, El Bahh B, Sundstrom L, Rougier A. Granule cell dispersion is correlated with early epileptic events in human temporal lobe epilepsy. J. Neurol. Sci., 154, 133-136 (1998).

29) Riban V, Bouilleret V, Pham-LêBT, L, Fritschy JM, Marescaux C, Depaulis A. Evolution of hippocampal epileptic activity during the development of hippocampal sclerosis in a mouse model of temporal lobe epilepsy. Neuroscience, 112, 101-111 (2002).

30) Parent JM, Murphy GG. Mechanisms and functional significance of aberrant seizure-induced hippocampal neurogenesis. Epilepsia, 49 (Suppl. 5), 19-25 (2008).

31) Scharfman H, Goodman J, McCloskey D. Ectopic granule cells of the rat dentate gyrus. Dev. Neurosci., 29, 14-27 (2007).

32) Muramatsu R, Ikegaya Y, Matsuki N, Koyama R. Early-life status epilepticus induces ectopic granule cells in adult mice dentate gyrus. Exp. Neurol., 211, 503-510 (2008).

33) Scharfman HE, Sollas AL, Goodman JH. Spontaneous recurrent seizures after pilocarpine-induced status epilepticus activate calbindin-immunoreactive hilar cells of the rat dentate gyrus. Neuroscience, 111, 71-81 (2002).

34) Scharfman HE, Goodman JH, Sollas AL. Granule-like neurons at the hilar/CA3 border after status epilepticus and their synchrony with area CA3 pyramidal cells: functional implications of seizureinduced neurogenesis. J. Neurosci., 20, 6144-6158 (2000).

35) Bell B, Lin JJ, Seidenberg M, Hermann B. The neurobiology of cognitive disorders in temporal lobe epilepsy. Nat. Rev. Neurol., 7 , 154-164 (2011).

36) Myers CE, Bermudez-Hernandez K, Scharfman HE. The influence of ectopic migration of granule cells into the hilus on dentate gyrus-CA3 function. PLoS ONE, 8, e68208 (2013).

37) Tao K, Ichikawa J, Matsuki N, Ikegaya Y, Koyama R. Experimental febrile seizures induce age-dependent structural plasticity and improve memory in mice. Neuroscience, 318, 34-44 (2016).

38) Koyama R, Tao K, Sasaki T, Ichikawa J, Miyamoto D, Muramatsu R, Matsuki N, Ikegaya Y. GABAergic excitation after febrile seizures induces ectopic granule cells and adult epilepsy. Nat. Med., 18, 1271-1278 (2012)

39) Koyama R. The use of organotypic slice cultures for the study of epileptogenesis. Neuropathology, 33, 475-479 (2013).

40) Koyama R, Matsuki N. Novel etiological and therapeutic strategies for neurodiseases: mechanisms and consequences of febrile seizures: lessons from animal models. J. Pharmacol. Sci., 113, 14-22 (2010).

41) Muramatsu R, Ikegaya Y, Matsuki N, Koyama R. Neonatally born granule cells numerically dominate adult mice dentate gyrus. $\mathrm{Neu}$ roscience, 148, 593-598 (2007).

42) Sajo M, Sugiyama H, Yamamoto H, Tanii T, Matsuki N, Ikegaya Y, Koyama R. Neuraminidase-dependent degradation of polysialic acid is required for the lamination of newly generated neurons. PLOS ONE, 11, e0146398 (2016)

43) Rakhade SN, Jensen FE. Epileptogenesis in the immature brain: 
emerging mechanisms. Nat. Rev. Neurol., 5, 380-391 (2009).

44) Ben-Ari Y. Excitatory actions of gaba during development: the nature of the nurture. Nat. Rev. Neurosci., 3, 728-739 (2002).

45) Löscher W, Klitgaard H, Twyman RE, Schmidt D. New avenues for anti-epileptic drug discovery and development. Nat. Rev. Drug Discov., 12, 757-776 (2013).

46) Löscher W, Puskarjov M, Kaila K. Cation-chloride cotransporters NKCC1 and KCC2 as potential targets for novel antiepileptic and antiepileptogenic treatments. Neuropharmacology, 69, 62-74 (2013).

47) Dzhala VI, Talos DM, Sdrulla DA, Brumback AC, Mathews GC, Benke TA, Delpire E, Jensen FE, Staley KJ. NKCC1 transporter facilitates seizures in the developing brain. Nat. Med., 11, 1205-1213 (2005).

48) $\overline{\text { Brandt }}$ C, Nozadze M, Heuchert N, Rattka M, Loscher W. Diseasemodifying effects of phenobarbital and the NKCC1 inhibitor bumetanide in the pilocarpine model of temporal lobe epilepsy. $J$. Neurosci., 30, 8602-8612 (2010).
49) Eftekhari S, Mehvari Habibabadi J, Najafi Ziarani M, Hashemi Fesharaki SS, Gharakhani M, Mostafavi H, Joghataei MT, Beladimoghadam N, Rahimian E, Hadjighassem MR. Bumetanide reduces seizure frequency in patients with temporal lobe epilepsy. Epilepsia, 54, e9-e12 (2013).

50) Ben-Ari Y, Tyzio R. Is it safe to use a diuretic to treat seizures early in development? Epilepsy Curr., 11, 192-195 (2011).

51) Sillanpää M, Schmidt D. Natural history of treated childhood-onset epilepsy: prospective, long-term population-based study. Brain, $\mathbf{1 2 9}$, 617-624 (2006)

52) Brodie MJ, Barry SJ, Bamagous GA, Norrie JD, Kwan P. Patterns of treatment response in newly diagnosed epilepsy. Neurology, $\mathbf{7 8}$, 1548-1554 (2012).

53) Devinsky O, Vezzani A, Najjar S, De Lanerolle NC, Rogawski MA. Glia and epilepsy: excitability and inflammation. Trends Neurosci., 36, 174-184 (2013). 